Revista Eletrônica em Gestão, Educação e Tecnologia Ambiental Santa Maria, v. 20, n. 1, jan.-abr. 2016, p. 372-380

Revista do Centro de Ciências Naturais e Exatas - UFSM

ISSN : 22361170

\title{
Dinâmica do uso e cobertura do solo no vale do Rio Pardo, Rio Grande do Sul
}

\author{
Dynamics of use and land cover in the Rio Pardo valley, Rio Grande do Sul
}

\begin{abstract}
Thomas Schroder, Rudiney Soares Pereira, Anna Paula Lora Zimmermann, Cristina Gouvea Redin, Marciane Danniela Fleck, Leonardo Mortari Machado e Nájila Souza Rocha
\end{abstract}

Universidade Federal de Santa Maria , Brasil

\begin{abstract}
Resumo
Os objetivos deste trabalho são determinar taxas de desmatamento e regeneração reais no período entre 1990 e 2014 e estimar mapas de uso e cobertura do solo para o ano de 2018 na região do Vale do Rio Pardo. Foram utilizadas imagens Landsat para a classificação do uso e cobertura do solo e o programa Dinamica EGO foi utilizado para determinar transições reais e estimadas entre as diferentes classes de uso do solo. A área florestal aumentou constantemente à uma taxa de $0,58 \%$ ao ano. No entanto, as taxas de desmatamento e regeneração são maiores, entre 3 e 6\%. O modelo desenvolvido gerou estimativas consistentes ao longo do período estudado. O mapa de uso do solo estimado para 2018 indica regiões suscetíveis ao desmatamento que devem ser melhor observadas pelos órgãos de fiscalização.
\end{abstract}

Palavras-chave: Dinamica Ego. Sensoriamento Remoto. Desmatamento. Predições Futuras. Floresta Nativa.

\begin{abstract}
The aims of this study were to evaluate rates of deforestation and forest regeneration in the time span between 1990 and 2014, furthermore to estimate land cover and use by the year of 2018 in the Rio Pardo Valley region. Landsat images were used for land cover and use classification and Dinamica EGO software was used to provide real and estimated transitions among land cover and use classes. Forested area increased at a rate of $0.58 \%$ by year. However, the rates of deforestation and forest regeneration are larger, between 3 and $6 \%$. The model developed showed to be consistent throughout the study time span. The land cover and use map estimated for 2018 shows regions which are susceptible to deforestation and should be closely watched by local authorities.
\end{abstract}

Keywords: Dinamica Ego. Remote Sensing. Deforestation. Future predictions. Native forest. 


\section{Introdução}

A Floresta Atlântica é conhecida internacionalmente por sua grande riqueza de espécies, das quais muitas são endêmicas (LEAL; CÂMARA, 2003; MYERS et al., 2000). Apesar de sua extensão original cobrir toda a parte leste do território brasileiro, restam apenas $11 \%$ da sua extensão original (RIBEIRO et al., 2009). No entanto existem discrepâncias entre diferentes estimativas, devido aos erros inerentes aos processos de estimativa, diferentes tamanhos de fragmentos que podem ser incluídos na análise e áreas de regeneração que podem ser excluídas, uma vez que não caracterizam a floresta original (SOS MATA ATLÂNTICA; INSTITUTO NACIONAL DE PESQUISAS ESPACIAIS, 2008).

No estado do Rio Grande do Sul (RS), o Inventário Florestal Contínuo chegou a conclusão que a área florestada do estado cresceu de 6\% em 1983 para 17\% em 2001 (RIO GRANDE DO SUL, 2002).Acredita-se que este aumento na área florestal está relacionado com o abandono de áreas agrícolas, devido ao grande uso de máquinas na agricultura fato que diminuiu a acessibilidade às áreas mais declivosas (BALBINO et al., 2011), expansão da silvicultura (DA SILVA, 2012) e, à uma maior fiscalização por parte de órgãos governamentais responsáveis pela aplicação das leis ambientais (BACKES, 2012). Além disso, deve-se ressaltar que existe uma sociedade mais 'verde', em defesa de valores ambientais do que há três décadas.

Embora haja uma recuperação da área de florestas no RS é necessário ressaltar que ainda existe desmatamento. Assim sendo, estimativas baseadas apenas na área florestal total tendem a subestimar o desmatamento e a recuperação das florestas no estado. Além de se determinar estas taxas, é necessário identificar áreas com maior risco de desmatamento, de forma a facilitar a fiscalização ambiental e a identificação de áreas para a conservação ambiental.

São várias as ferramentas disponíveis para a modelagem espaço - temporal de mudança de uso e cobertura do solo (PERRY; ENRIGHT, 2006). Este pode ser baseado em estimativas através de autômatos celulares como o Dinamica EGO (SOARES-FILHO; CERQUEIRA; PENNACHIN, 2002) e LANDIS (MLADENOFF, 2004), ou em simulações de Monte Carlo, usando Cadeia de Markov, como o StocModLCC (ROSA et al., 2013). O Dinamica EGO destaca-se como um programa com mais tempo de desenvolvimento, desta forma apresenta menor probabilidade de erros, flexibilidade e velocidade na modelagem. Além disso, este software já demonstrou sua utilidade na análise e modelagem de mudanças de uso e cobertura do solo no RS (BENEDETTI, 2010; FERRARI, 2008; FURLAN, 2008).

Assim sendo, os objetivos deste trabalho são: Identificação de taxas de desmatamento e regeneração na região do Vale do Rio Pardo no RS, durante um período de 24 anos entre os anos 1990 e 2014, e um mapa com prognóstico de uso e cobertura do solo para esta mesma região no ano de 2018.

\section{Metodologia}

A área de estudo corresponde à um recorte retangular de $1376,63 \mathrm{Km}^{2}$ compreendido entre as

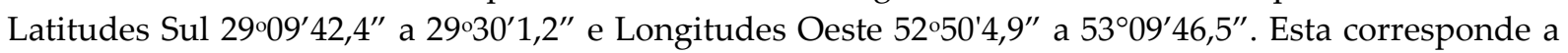
uma área de transição entre a Floresta Estacional Decidual e a Floresta Ombrófila Mista (RIO GRANDE DO SUL, 2002). Por corresponder a uma área relativamente extensa, existe uma variação edáfica muito grande, enquanto que o clima da região é classificado como Cfa de acordo com Köppen (ALVARES et al., 2013).

Foram utilizadas imagens dos satélites Landsat 5 (anos de 1990, 1995, 2000, 2005 e 2010) e Landsat 8 (ano de 2014), todas possuindo uma resolução espacial de 30 metros. Objetivou-se obter imagens do mês de fevereiro dos anos pré-determinados, no entanto, caso existisse cobertura de nuvens na imagem obtida neste período, optou-se por utilizar imagens mais limpas de um período próximo. O datum WGS 84 (World Geodetic System) foi utilizado em todas as análises destas imagens. 
Realizou-se a classificação supervisionada destas imagens por meio do software SPRING, desenvolvido pelo Instituto Nacional de Pesquisas Espaciais (INPE). Foram construídas composições de cor natural com correção do histograma de frequência para todos os períodos analisados e especificadas três classes de uso da terra a priori: Floresta, Agropecuária e Áreas Urbanas. Teoricamente é possível dividir áreas de pecuária e agricultura, no entanto, frente à indisponibilidade de imagens de melhor qualidade de todos os anos para aquisição, esta divisão torna-se mais complexa. Também é possível acrescentar uma classe relacionada a corpos de água, mas esta representa apenas 0,6\% da área (PEREIRA, 2013), soma-se ainda o fato de ser ineficaz a classificação desta classe para imagens com a resolução espacial utilizadas neste estudo.

Para a construção de um mapa categórico de classes de declividade, foram utilizados mapas de hidrografia e estrutura viária obtidos da Secretária Estadual do Meio Ambiente (SEMA). Os mapas foram elaborados por meio de imagens ASTER (Advanced Spaceborne Thermal Emission and Reflection Radiometer). Este último mapa é caracterizado por 6 classes equidistantes de declividade em graus, variando de 0 a $60^{\circ}$ e correspondem à características naturais da área que estão relacionadas com causas do desmatamento e regeneração florestal (BENEDETTI, 2010).

O Dinamica EGO (Environment for Geoprocessing Objects) pode ser visto como um ambiente analítico - heurístico para calibração de modelos de mudança de uso e cobertura do solo (SOARESFILHO; RODRIGUES; FOLLADOR, 2013), que foi desenvolvido inicialmente para a modelagem do desmatamento na Amazônia brasileira (SOARES-FILHO; RODRIGUES; COSTA, 2009). De acordo com Burrough e McDonnel (1998) e Benedetti (2010), os modelos dinâmicos em um ambiente SIG visam realizar a simulação numérica de processos dependentes do tempo.

Este programa baseia-se no uso de pesos de evidência para avaliar a influência de variáveis estáticas e dinâmicas na mudança do uso do solo. As probabilidades de mudança são calculadas através de uma matriz de transição que pode ser decomposta em passos menores que a distância temporal entre dois mapas categóricos de classificação através das propriedades de matrizes Markovianas de transição (SOARES-FILHO; CERQUEIRA; PENNACHIN, 2002).

As estimativas são geradas através de dois algoritmos deste programa: O Patcher e o Expander. O último se refere à modelagem da expansão de fragmentos das classes existentes no passo inicial, enquanto o Patcher é responsável pela criação de novos fragmentos. A validação dos resultados é baseado no método fuzzy (SOARES-FILHO; RODRIGUES; COSTA, 2009), que avalia acertos de transição exatos e transições reais que ocorrem nas proximidades de transições estimadas. O método fuzzy permite avaliar a qualidade da calibração do modelo (proporção entre Patcher e Expander, e tamanho dos fragmentos gerados), além disso, foram realizadas comparações visuais entre os mapas de classificação observados e estimados. A partir da calibração destes parâmetros no modelo foram gerados mapas de uso e cobertura do solo para o ano de 2018.

\section{Resultados e discussões}

Uma análise comparando a área de florestas no período inicial (1990) e final da avaliação (2014), mostra que a cobertura florestal cresceu de $343,4 \mathrm{Km}^{2}(24,9 \%)$ para $520,3 \mathrm{Km}^{2}(37,8 \%)$, correspondendo à um crescimento anual de $0,58 \%\left(7,98 \mathrm{Km}^{2} / \mathrm{ano}\right)$ de cobertura florestal por ano. Estes resultados de cobertura florestal para 2014 são semelhantes aos obtidos com imagens RapidEye (PEREIRA, 2013), que possuem resolução espacial 36 vezes melhor que as imagens utilizadas neste estudo, o que corrobora com a qualidade dos resultados aqui apresentados.

A análise multitemporal mostra que as variações de uso e cobertura do solo são muito mais acentuadas. A Figura 1 demonstra que as taxas de desmatamento e regeneração são muito maiores do que o simples aumento da cobertura florestal. As taxas variam de 3 até $6 \%$ da área total sob estudo (41,3 até $\left.82,6 \mathrm{Km}^{2} / \mathrm{ano}\right)$.

Desta forma, mesmo havendo um aumento da cobertura florestal ao longo do tempo, ocorrem ainda quantidades significativas de desmatamento, problema a ser combatido na área em estudo e em todo o domínio da Floresta Atlântica. Este fato, deve-se principalmente devido à pressão antrópica,

REGET - V. 20, n. 1, jan.- abr. 2016, p.372-380 
estimulada principalmente pelas atividades inerentes à região de estudo, tais como agricultura e pecuária extensiva. Em estudo feito por Evangelista (2010) em áreas do Bioma Pampa, foi detectado por meio de construção de cenários de tendência, a diminuição das áreas de vegetação natural em detrimento à outras atividades, principalmente para o cultivo de soja e silvicultura.

Em contrapartida, Ferrari (2008), evidenciou um aumento de $2 \%$ da área florestal na região da Quarta Colônia, também no RS, entre o período de 1988 até 2002. Já para o período estipulado entre os anos de 2002 até 2008, o autor não encontrou resultados significativos.

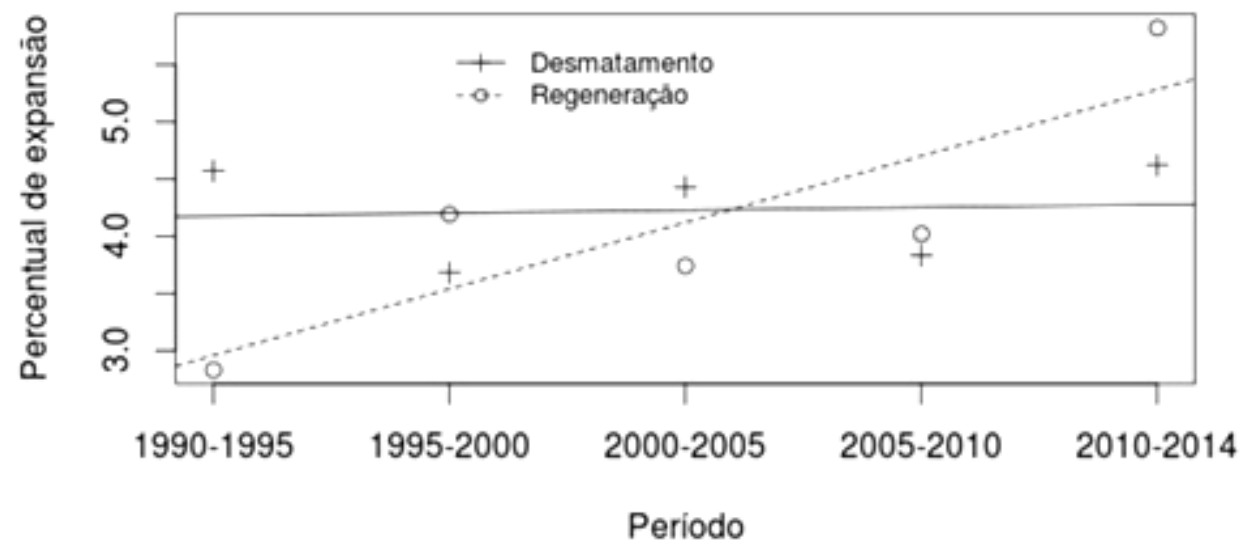

Figura 1 - Taxas anuais de desmatamento e regeneração florestal no Vale do Rio Pardo - RS. As linhas representam tendências lineares para cada uma das classes de mudança de uso do solo.

Nem todos os coeficientes estimados foram significativos, mas todos foram mantidos no modelo por representarem relações comuns em processos de mudança da cobertura do solo (ROSA et al., 2014; WALKER et al., 2013). A maioria dos coeficientes estimados para as variáveis dinâmicas e estáticas mostraram um padrão congruente ao longo do tempo. As florestas expandiram em áreas de maior declividade como esperado. Conforme Rovedder (2013) o componente florestal está presente em afloramentos rochosos, margens de mananciais hídricos e encostas declivosas no RS.

Outro comportamento esperado e confirmado foi o de maior probabilidade de regeneração em áreas próximas às florestas e maior desmatamento próximo a áreas de agropecuária, demonstrando que o desmatamento depende do acesso às áreas florestais para o transporte da madeira. Alencar et al. (2004) cita a abertura de estradas ilegais em áreas da Amazônia como principal fator vinculado ao desmatamento de florestas intactas. Da mesma forma, a regeneração florestal depende da disponibilidade de sementes para o estabelecimento da vegetação, assim sendo, áreas próximas às florestas têm maior tendência à regeneração, devido às fontes de propágulo.

Uma comparação transversal (nos mesmos anos) entre os mapas mostra uma leve tendência de superestimativa dos fragmentos gerados pelo algoritmo (Figura 2). No entanto uma análise transversal (entre anos) demonstra a capacidade do mesmo em predizer áreas sob forte pressão de desmatamento e locais sujeitos à regeneração. Estas diferenças são normais em qualquer processo de modelagem, uma vez que os processos reais envolvem muitas variáveis que são não - observáveis e variáveis que são muito caras de se obter.

Outros efeitos sobre a dinâmica do uso da terra desta região que poderiam ser importantes são: Valor de mercado da produção agrícola corrigida pela inflação no espaço - tempo sob estudo, 
variação entre área plantada de diferentes culturas no espaço - tempo e incentivos econômicos para a silvicultura.
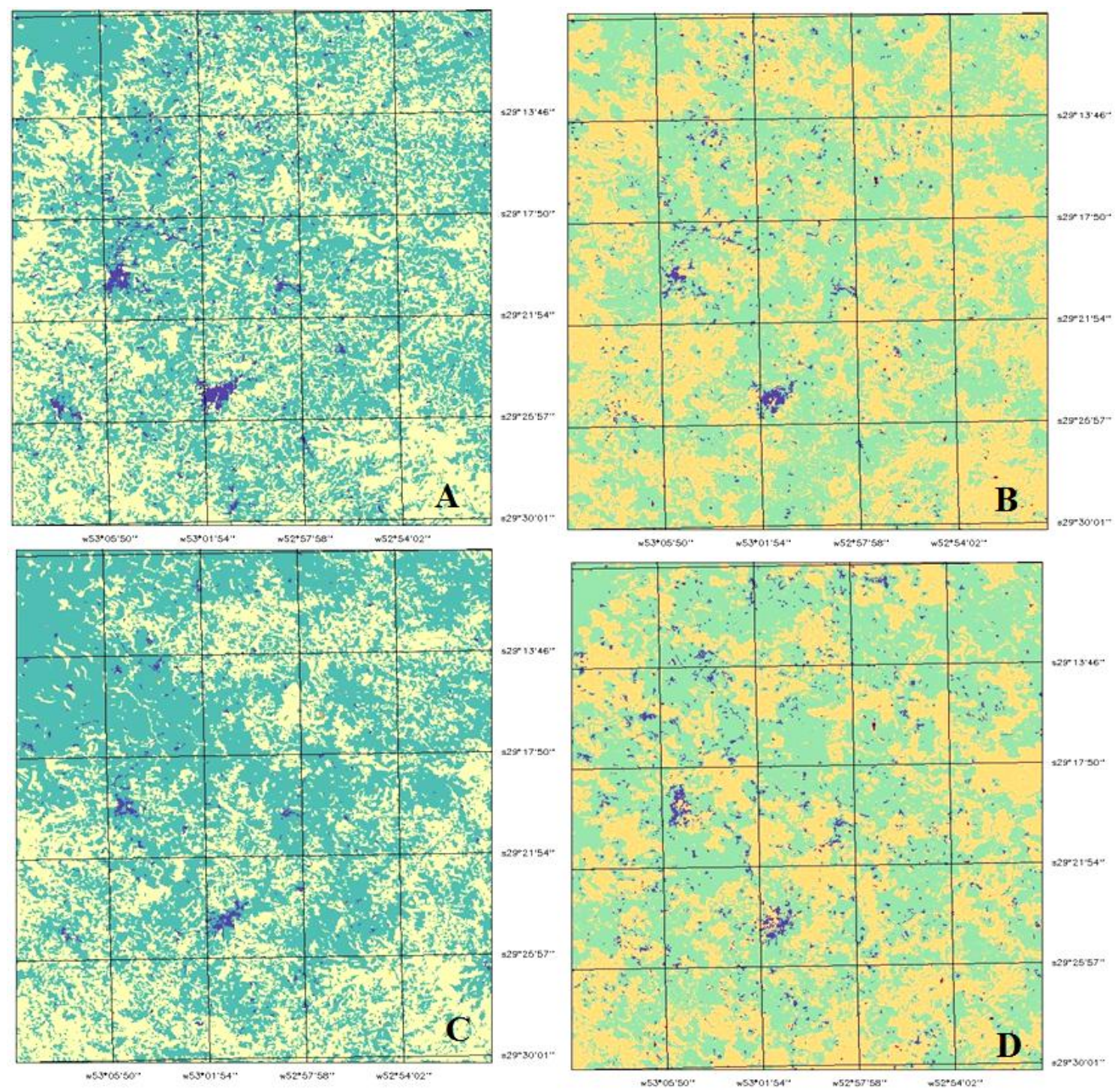

Figura 2 - Mapas categóricos de classificação do uso da terra para a Região do Vale do Rio Pardo - RS, sendo: 1990 predito (A) e observado (B) e 2014 predito (C) e observado (D). Os mapas preditos correspondem às simulações realizadas pelo programa Dinamica EGO, enquanto os mapas observados correspondem a classificação realizada com imagens Landsat do mesmo período. As regiões em verde correspondem a florestas, áreas em amarelo correspondem à agropecuária.

A análise pelo método Fuzzy destaca a eficiência do modelo produzido (Tabela 1). Este método pode ser utilizado em diferentes áreas de resolução, no entanto, janelas de resolução de acima de 11 pixels se mostram ineficientes (BENEDETTI, 2010). Os resultados demonstram que entre 45 e $53 \%$ das estimativas de mudança de uso do solo foram exatas, ou seja, ocorreram de forma igual às transições preditas pelo modelo. As similaridades para a janelas de 11 x 11 variaram entre 88 e 95\%, indicando que esta proporção de transições reais ocorreu em menos 150 metros de distância de onde foram preditas transições do mesmo tipo. Estes valores altos de similaridade em distâncias tão pequenas em relação à área de estudo, demonstram a alta qualidade do modelo. 
Tabela 1 - Similaridade pelo método Fuzzy para diferentes tamanhos de janela para o modelo de dinâmica de uso e cobertura do solo desenvolvido através do programa Dinamica EGO, para a região do Vale do Rio Pardo - RS

\begin{tabular}{ccc}
\hline \multirow{2}{*}{ Período } & $\begin{array}{c}\text { Tamanho da janela } \\
\text { (Pixels) }\end{array}$ & Similaridade (Fuzzy) \\
\hline \multirow{3}{*}{$1990-1995$} & $1 \times 1$ & 0,458 \\
\cline { 2 - 3 } & $3 \times 3$ & 0,622 \\
\cline { 2 - 3 } 1995-2000 & $11 \times 11$ & 0,881 \\
\cline { 2 - 3 } & $1 \times 1$ & 0,449 \\
\cline { 2 - 3 } & $3 \times 3$ & 0,634 \\
\hline \multirow{2}{*}{$2000-2005$} & $11 \times 11$ & 0,921 \\
\cline { 2 - 3 } & $1 \times 1$ & 0,512 \\
\cline { 2 - 3 } $2005-2010$ & $3 \times 3$ & 0,681 \\
\cline { 2 - 3 } & $11 \times 11$ & 0,928 \\
\cline { 2 - 3 } & $1 \times 1$ & 0,451 \\
\hline \multirow{2}{*}{$2010-2014$} & $3 \times 3$ & 0,624 \\
\cline { 2 - 3 } & $11 \times 11$ & 0,915 \\
\cline { 2 - 3 } & $1 \times 1$ & 0,537 \\
\hline
\end{tabular}

À partir do modelo desenvolvido é possível estimar as classes de uso do solo no ano de 2018 à partir do mapa observado em 2014 (Figura 3). O mapa indica que existe uma suscetibilidade ao desmatamento nas regiões centro - leste e noroeste da região em estudo. Estas são indicações que podem colaborar com a fiscalização de órgãos ambientais contra o desmatamento. Entretanto, o mapa predito tem uma proporção maior ( $2 \%$ da área total) de floresta que a situação observada no ano de 2014. Desta forma, espera-se que a tendência de aumento da área coberta por florestas continue a se manter pelos próximos anos. 


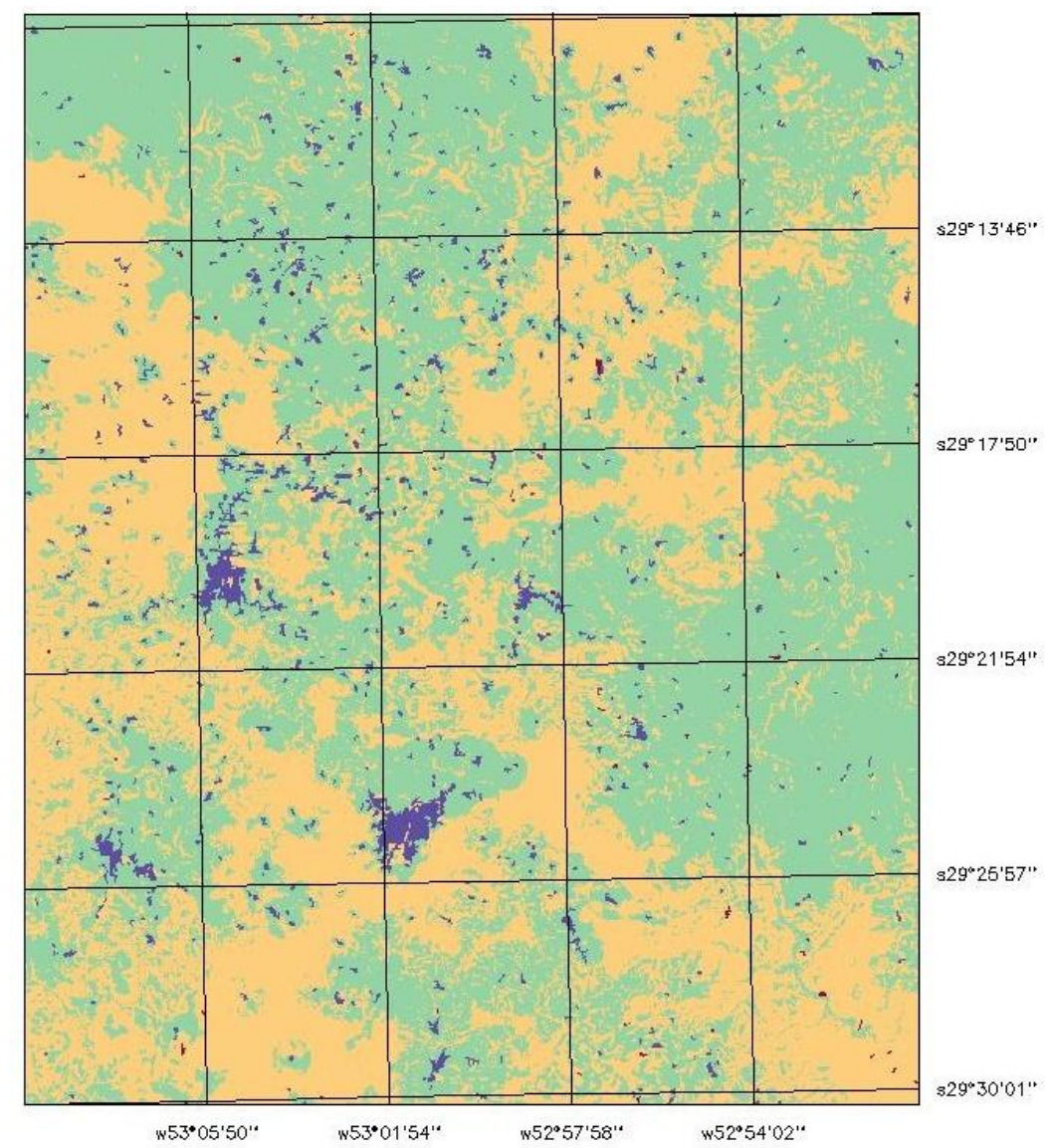

Figura 3. Mapa categórico estimado de uso e cobertura do solo pelo modelo desenvolvido através do programa Dinamica EGO para o ano de 2018, para a região do Vale do Rio Pardo - RS. As regiões em verde correspondem às florestas, áreas em amarelo correspondem à agropecuária e, áreas em vermelho são cidades.

\section{Conclusões}

Ocorreu aumento da cobertura florestal no Vale do Rio Pardo - RS, com taxa de 0,58\% da área ao ano; Este aumento é devido a diferença entre processos (desmatamento e regeneração) muito mais intensos (entre 3 e 6\% da área total);

O modelo desenvolvido apresentou boa capacidade de predição da dinâmica de uso e cobertura do solo;

O mapa estimado para 2018 indica áreas que estão sujeitas ao desmatamento e permite uma melhor fiscalização da região;

A tendência de aumento de área florestal deve continuar. 


\section{REFERÊNCIAS}

ALENCAR, A.; NEPSTAD, D.; MCGRATH, D.; MOUTINHO, P.; PACHECO. P.; DIAZ, M.D.C.V; SOARES- FILHO, B. Desmatamento na Amazônia: indo além da "Emergência Crônica". Instituto de Pesquisas Ambiental da Amazônia. Belém. 85pp. 2004.

ALVARES CA et al. Köppen's climate classification map for Brazil. Meteorologische Zeitschrift, v. 22, n. 6, p. 711-728, 2013.

BACKES A. Áreas protegidas no estado do Rio Grande do Sul: esforço para a conservação. Pesquisas Botânica, v. 63, p. 225-355, 2012.

BALBINO LC et al. Evolução tecnológica e arranjos produtivos de sistemas de integração lavourapecuária-floresta no Brasil. Pesquisa Agropecuária Brasileira, v. 46, n. 1, p. 1-12, 2011.

BENEDETTI ACP. Modelagem dinâmica para simulação de mudanças na cobertura florestal das serras do sudeste e campanha meridional do Rio Grande do Sul. [s.l.] Universidade Federal de Santa Maria, 2010.

BURROUGH,PA.; MCDONNEL, RA. Principles of geographical information systems. Oxford: Clarendon Press, 333p. 1998.

DA SILVA MD. Os cultivos florestais do pampa, no sul do Rio Grande do Sul: Desafios, perdas e perspectivas frente ao avanço de novas fronteiras agrícolas. Floresta, v. 42, p. 215-226, 2012.

EVANGELISTA, FM. Construção de cenários para estimativa dos impactos das atividades socioeconômicas sobre a vegetação do Bioma Pampa. [s.l.] Universidade Federal do Rio Grande do Sul, 2010.

FERRARI R. Modelagem Dinâmica do Uso e Cobertura da Terra da Quarta Colônia, RS. [s.l.] Universidade Federal de Santa Maria, 2008.

FURLAN MC. Modelagem dinâmica de uso e cobertura da terra da bacia de Arroio Grande - RS. [s.l.] Universidade Federal de Santa Maria, 2008.

LEAL CG, Câmara I. The Atlantic Forest of South America: biodiversity status, threats, and outlook. [s.l.] Island Press, v. 1, 2003.

MLADENOFF DJ. Landis and forest landscape models. Ecological Modelling, v. 180, n. January, p. 7$19,2004$.

MYERS N. et al. Biodiversity Hotspots for Conservation Priorities Nature, 2000.

PEREIRA RS. Tecnologia de Monitoramento Automatizado da Cobertura Florestal em Áreas de Floresta Estacional Decidual na Região Centro-Serra do RS. Santa Maria: [2013].

PERRY GLW, ENRIGHT NJ. Spatial modelling of vegetation change in dynamic landscapes: a review of methods and applications. Progress in Physical Geography, v. 30, p. 47-72, 2006. 
RIBEIRO MC. et al. The Brazilian Atlantic Forest: How much is left, and how is the remaining forest distributed? Implications for conservation. Biological Conservation, v. 142, n. 6, p. 1141-1153, 2009.

RIO GRANDE DO SUL. Secretaria Estadual do Meio Ambiente. Inventário florestal contínuo do Rio Grande do Sul. Santa Maria: SEMA/UFSM, 2002.

ROSA IMD. et al. Predictive Modelling of Contagious Deforestation in the Brazilian Amazon. PLoS ONE, v. 8, n. 10, 2013.

ROSA IMD. et al. Modelling land cover change in the Brazilian Amazon: temporal changes in drivers and calibration issues. Regional Environmental Change, p. 123-137, 2014.

ROVEDDER, APM. Bioma Pampa: Relacões solo-vegetação e experiências de restauração. In: CONGRESSO NACIONAL DE BOTÂNICA, 64ํㅜ 2013, Belo Horizonte, Anais... Belo Horizonte: Sociedade Botânica do Brasil, 2013, p. 46-53.

SOARES-FILHO B, RODRIGUES H, COSTA W. Modeling environmental dynamics with Dinamica EGO. Belo Horizonte: [s.n.]. p. 114, 2009.

SOARES-FILHO B, RODRIGUES H, FOLLADOR M. A hybrid analytical-heuristic method for calibrating land-use change models. Environmental Modelling and Software, v. 43, p. 80-87, 2013.

SOARES-FILHO BS, CERQUEIRA GC, PENNACHIN CL. DINAMICA - A stochastic cellular automata model designed to simulate the landscape dynamics in an Amazonian colonization frontier. Ecological Modelling, v. 154, p. 217-235, 2002.

SOS MATA ATLÂNTICA; Instituto Nacional de Pesquisas Espaciais. Atlas dos remanescentes florestais da Mata Atlântica, período de 2000 a 2005. [s.l: s.n.], 2006.

WALKER $\mathrm{R}$ et al. Modeling spatial decisions with graph theory: Logging roads and forest fragmentation in the Brazilian Amazon. Ecological Applications, v. 23, n. 1, p. 239-254, 2013. 